\title{
Evaluation of Usage fMRI in Alzheimer's Disease Diagnose
}

\author{
Sohaila Yazdani, ${ }^{1,}$ Karim Khoshgard, ${ }^{1}$ and Hasan Norouzi ${ }^{1}$ \\ ${ }^{1}$ Kermanshah University of Medical Sciences, Kermanshah, Iran \\ "Corresponding author: Sohaila Yazdani, Kermanshah University of Medical Sciences, Kermanshah, Iran. E-mail: sohailayazdani@gmail.com
}

Received 2016 December 21; Accepted 2017 February 08.

\begin{abstract}
Background: Alzheimer's disease (AD) is a state where neurons within the brain stop functioning, lose connection with other neurons and die. It's the most common cause of dementia, a loss of brain function that can harmfully impact memory, thinking, language, judgment and behavior. Alzheimer's is irreversible and progressive. Although the cause of Alzheimer's disease is unknown, scientists believe that a build-up of beta-amyloid plaques and neurofibrillary tangles in the brain are associated with the disease. Medications that slow the progression of the disease and manage symptoms are available, but there is no cure for Alzheimer's disease. Current diagnosis of AD is through clinical, neuropsychological, and neuroimaging assessments. Functional MRI (fMRI) measures brain activity during a cognitive, sensory, or motor task or at rest by measuring changes in blood oxygen level dependent (BOLD) MR signal.

Objectives: In this paper, we evaluate application of fMRI to detect AD.

Methods: The papers were searched in PubMed, Medline and Scopus databases with the relevant key words such as fMRI, Alzheimer disease and early detection.

Results: BOLD fMRI is considered to reflect the joined synaptic activity of neurons through MRI signal changes because of alterations in blood flow, blood volume, and the blood oxyhemoglobin/deoxyhemoglobin ratio. In patients with clinically diagnosed AD, the outcomes of fMRI have been quite consistent, showing reduced hippocampal activity during the encoding of new data. Several studies have reported increased prefrontal cortical activity in AD patients, suggesting that other networks may increase activity as an attempted compensatory mechanism during hippocampal failure. In particular, the event-related fMRI studies have found that hyperactivity was observed, which suggested that hyperactivity might represent a compensatory mechanism in the setting of early AD pathology. Also, fMRI of default mode network (DMN) brain activity during resting is lately achievement attention as a potential noninvasive biomarker to diagnose initial AD.

Conclusions: Both task-related and resting fMRI techniques have the potential to detect early brain dysfunction related to AD. However, the use of $\mathrm{fMRI}$ in $\mathrm{AD}$ populations thus far has been limited to a relatively small number of research groups.
\end{abstract}

Keywords: FMRI, Alzheimer Disease and Early Detection

This is an abstract presented in the 33rd Iranian congress of radiology (ICR) and the 15th congress of Iranian radiographic science association (IRSA). 